\title{
A reactive self-organised scheduling based on multi-agent paradigm
}

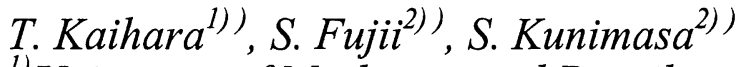 \\ ${ }^{1)}$ University of Marketing and Distribution Sciences, Faculty of \\ Information Science \\ 3-1, Gakuen-nishi, Nishi, Kobe 651-2188, Japan \\ e-mail:kaihara@umds.ac.jp \\ ${ }^{2)}$ Kobe University, Faculty of Engineering \\ Rokkodai, Nada, Kobe, 657-8501, Japan \\ e-mail:fujii@seg.kobe-u.ac.jp, \\ kunimasa@kaede.seg.kobe-u.ac.jp
}

\begin{abstract}
This paper denotes the abilities of multi-agent paradigm with reinforcement learning algorithm to obtain a sophisticated work movement in job shop type manufacturing scheduling problem. Several scheduling objectives are conducted by the social goal of the agents. Learning algorithm is introduced to improve the agent movements without losing their robustness. The well-educated work agents prove capable of robustness and reliability coping with major scheduling demands through several simulation scenarios.
\end{abstract}

\section{Keywords}

Manufacturing scheduling, Multi-agent, Reinforcement learning, Robustness

\section{INTRODUCTION}

As the size and complexity of manufacturing systems increase, it becomes difficult to get universal solutions in any conditions (Uzory, 1992). System robustness is a major concern especially in reactive scheduling system.

The best known of natural collective systems are social insects, such as bee or aunts: they use simple components; they use many identical insects; they exhibit system reliability; they adapt to environmental changes; they are robust with respect to individual insect failures (Langton, 1986). Scheduling systems which are modelled on such systems are expected to overcome current scheduling problems (Kaihara, 1997). Recently the utilisation of multi-agent system in manufacturing application increases (Deneubourg, 1991). Multi-agent paradigm has several characteristics, such as autonomy, pro-activeness, social ability, and reactivity, to 
overcome the current scheduling problems in large scaled manufacturing systems. These characteristics facilitate a scheduling algorithm based on coordinated motions of collective autonomous agents.

Reinforcement learning is a synthesis of a number of paradigms based on learning mechanism in nature (Grefenstette, 1988), and quite effective to the multiagent based scheduling paradigm, because agent knowledge on scheduling rule is reinforced as they acquire much more experience. Agent reactive movement will become sophisticated with robustness by the learning algorithm.

The dynamics of a multi-agent based scheduling paradigm with reinforcement learning is analysed in this paper. Our goal is to propose a decentralised universal scheduling concept and confirm its robustness against several non-linear disturbances in manufacturing system by using computer simulation model.

\section{SELF-ORGANISED SCHEDULING PARADIGM}

\subsection{Agent definition}

Each work existing in manufacturing system is defined as agent in our research. The simplest possible architecture that conducts self-organisation and solves unified goal is our research topics. We believe the system robustness is inducted by a number of agents which equip primitive architecture, such as simple sensor, simple mobility, minimal communication and computation (Kaihara, 1997).

\subsection{Cooperation algorithm in work agent}

Cooperation algorithm amongst agents is the most important item, which characterise the agent actions in the system (Colorni, 1992). Our major concern is to build up the simplest architecture with robustness, and cooperation amongst work agents is established through their minimal communication. In our previous research works (Kaihara, 1997, 1998), an entrainment algorithm was proposed for agent protocol of cooperation phenomenon in reactive scheduling problem.

The proposed entrainment algorithm has been proved to be robust enough to apply job-shop type manufacturing system. It, however, has been also clarified that the cooperation algorithm is not always concerned with scheduling optimisation.

\subsection{Reinforcement learning approach}

Reinforcement learning aims to adapt a system to a given environment according to rewards. Profit sharing method derives its behaviour from a metaphor of the learning processes in nature (Grefenstette, 1988). In this scheme, a rule sequence applied between rewards is called an episode, and problem solving is divided into episodes delimited by the receipt of external rewards. A rule is active during a given episode if it wins a bidding competition during any step of the episode. A function that shares the reward amongst rules is called a reinforcement function.

Profit sharing approach is applied into the agent based scheduling to reinforce work agents. The reinforcement function $f$ in this paper is defined as follows:

$$
f_{j}=(1 / S) f_{j-1} \quad \text { where } S: \text { Geometric ratio }
$$


$f_{j}$ : Reward or Penalty to $j$ th previous rule

Agent movement in profit sharing algorithm is shown in Figure 1. The episode of each agent is modified in cooperative movement. The number of rules in the agent memory buffer is limited within $R H$. The agent memory buffer holds the latest executed rules in cooperation movement. Weight allocation to the rules is executed by the reinforcement function shown in (1). Each agent keeps the average arrival time span between any two process machines. The agent gets the reward, a positive value, if it arrives at the target machine faster than the current average arrival time of itself. Otherwise the agent gets penalty, a negative value.

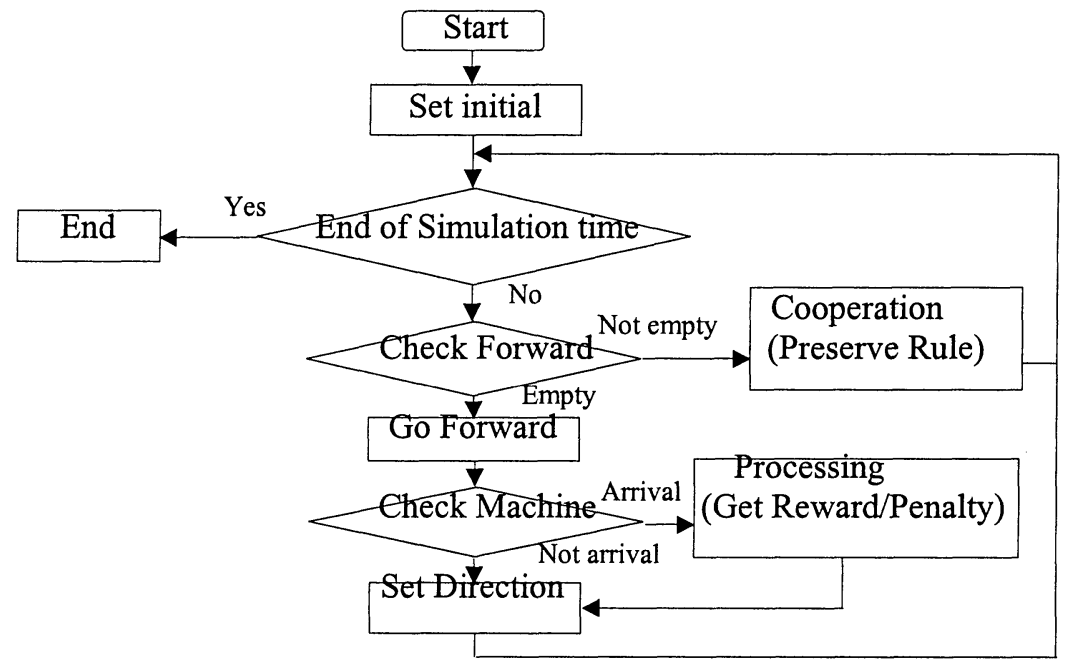

Figure 1 Flow chart in profit sharing

\subsection{Agent definition}

Rule information, which consists of condition part and action part, is shown in Figure 2. Each rule has 5 bit in length with weight, which is maintained by both rewards and penalty, and the first 3 bit is for the condition part, and the remained 2 bit represents the action part. A work agent holds 32 kinds of rules at maximum.

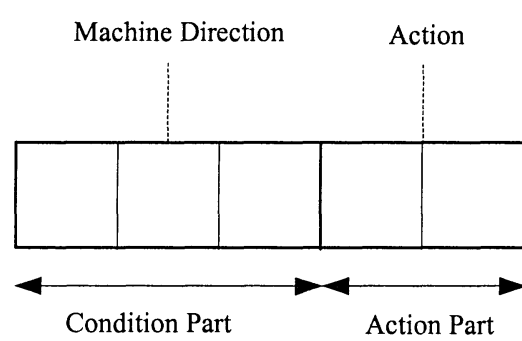

Figure 2 Rule information

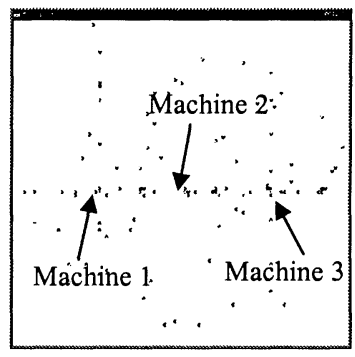

Figure 3 Experimental model 
Table 1 represents contained information in the rules. The direction to the target process machine is preserved in the condition part. For example, in case the target machine is located just in the moving direction, the condition part is ' 000 '. The action part indicates agent direction to move. The agents are allowed to move only in 4 directions due to the possible simplest moving mechanism. Each agent doesn't hold any rule initially in order to increase learning performance.

Table 1 Rule data

\begin{tabular}{ll|ll}
\hline $\begin{array}{l}\text { Condition } \\
\text { Part }\end{array}$ & Input & $\begin{array}{l}\text { Action } \\
\text { Part }\end{array}$ & Action \\
\hline 000 & Fore(Forward) & 00 & Keep Current \\
001 & Left & 01 & Turn Left \\
010 & Back & 10 & Turn Backward \\
011 & Right & 11 & Turn Right \\
100 & ForeLeft & & \\
101 & BackLeft & & \\
110 & BackRight & & \\
111 & ForeRight & & \\
\hline
\end{tabular}

\section{EXPERIMENTAL RESULTS}

Basic analysis of reactive self-organised scheduling with reinforcement learning approach is examined by using simulation model so as to investigate its effectiveness and robustness in dynamic conditions.

\subsection{Simulation model}

Figure 3 shows a factory model in our simulation experiment. The initial position $P$ of agent $i$ is randomly defined shown in this figure. Each agent is placed as an arrow that shows its current direction. The initial direction $D i_{0}$ is also randomly defined. Process machine is shown as small dots. Scheduling performance is estimated by process operation completion ratio $(O C R)$, defined as follows:

$$
O C R=\underset{i=1, W}{\Sigma_{F i}} / W
$$

where W: Total number of agents, $F i$ : The number of finished operations of agent $i$.

\subsection{Simulation parameters}

Default simulation parameters are defines as follows:

$$
W=60, M=3, N i=3, T T Y=2
$$

where

M: $\quad$ Total number of process machines, TTY: Total number of agent type

$N i$ : Total number of operations of agent $i$

Followings are the process machine sequence definitions: 
If $\left({ }^{\exists} A g t_{i t}(T Y i=1)=\right.$ True $) \quad$ then $P M o_{i 1}=1, P M o_{i 2}=2, P M o_{i 3}=3$

If $\left({ }^{A} A g t_{i t}\left(T_{i}=2\right)=\right.$ True $) \quad$ then $P M o_{i 1}=3, P M o_{i 2}=2, P M o_{i 3}=1$

where

TYi: Type of agent $i, O i j$ : Operation index $j$ of agent $i, P M o_{i j}$ : Machine index of Oij

Parameters concerned with profit sharing approach are shown in Table 2.

Table 2 Parameters of profit sharing

\begin{tabular}{ll}
\hline \multicolumn{1}{c}{ RH } & 10 \\
\hline$S$ & 2 \\
Reward & Type A: 0.01 \\
& Type B: 0.1 \\
& Type C: 1.0 \\
Penalty & Type A: 0.01 \\
& Type B: 0.1 \\
& Type C: 1.0 \\
\hline
\end{tabular}

Three types of constant values both in reward and in penalty are examined to investigate reasonable credit assignment for the proposed reactive scheduling.

\subsection{Simulation results}

Scheduling performance of several experimental conditions are compared in Figure 4. 'Normal' means conventional self-organised reactive scheduling approach without any learning algorithm. Cooperation movement is based on the entrainment algorithm. While reinforcement learning approach enhances scheduling performance in type $\mathrm{B}$, it decreases the performance in type $\mathrm{C}$.

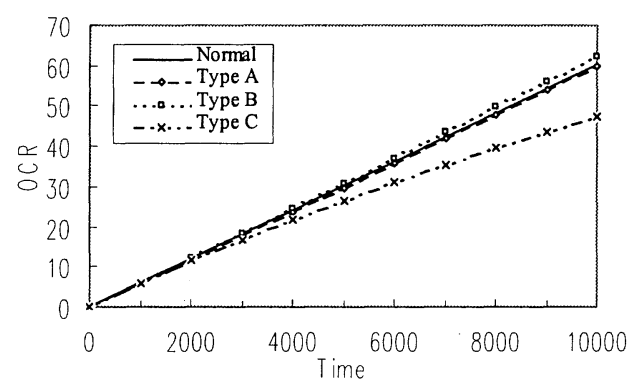

Figure 4 Scheduling performance

Final weight values of all the rules in all the agents were analysed to investigate the profit sharing effects on the self-organised scheduling approach. Table 3 (a) and (b) show the final average weight values of each rule in type B and C, respectively. 
Table 3 (a) Acquired weight in rules (Type B)

\begin{tabular}{cccccc}
\hline & 00 & 01 & 10 & 11 & AVEDEV \\
\hline 000 & 113.01 & 72.48 & 15.7 & 90.75 & \\
& $38.7 \%$ & $24.8 \%$ & $5.4 \%$ & $31.1 \%$ & 0.099 \\
001 & 4.12 & 10.12 & 5.82 & 2.63 & \\
& $18.2 \%$ & $44.6 \%$ & $25.7 \%$ & $11.6 \%$ & 0.101 \\
010 & 1.59 & 7.58 & 4.17 & 5.02 & \\
& $8.7 \%$ & $41.3 \%$ & $22.7 \%$ & $27.3 \%$ & 0.093 \\
011 & 2.44 & 2.59 & 3.96 & 8.07 & \\
& $14.3 \%$ & $15.2 \%$ & $23.2 \%$ & $47.3 \%$ & 0.112 \\
100 & 19.91 & 88.38 & 47.98 & 39.13 & \\
& $10.2 \%$ & $45.2 \%$ & $24.6 \%$ & $20.0 \%$ & 0.101 \\
101 & 2.35 & 5.91 & 3.71 & 4.69 & \\
& $14.1 \%$ & $35.5 \%$ & $22.3 \%$ & $28.2 \%$ & 0.068 \\
110 & 3.13 & 3.12 & 6.58 & 3.83 & \\
& $18.8 \%$ & $18.7 \%$ & $39.5 \%$ & $23.0 \%$ & 0.072 \\
111 & 34.8 & 35.99 & 93.17 & 68.84 & \\
& $14.9 \%$ & $15.5 \%$ & $40.0 \%$ & $29.6 \%$ & 0.098 \\
\hline
\end{tabular}

Table 3 (b) Acquired weight in rules (Type C)

\begin{tabular}{ccccccc}
\hline & 00 & OT & TO & I1 & AVEDEV \\
\hline W & 414.72 & 502.34 & 135.62 & 283.18 & \\
& $31.0 \%$ & $37.6 \%$ & $10.2 \%$ & $21.2 \%$ & 0.093 \\
001 & 10.92 & 14.6 & 22.93 & 8.98 & \\
& $19.0 \%$ & $25.4 \%$ & $39.9 \%$ & $15.6 \%$ & 0.077 \\
010 & 6.6 & 38.52 & 18.04 & 44.23 & \\
& $6.1 \%$ & $35.9 \%$ & $16.8 \%$ & $41.2 \%$ & 0.135 \\
011 & 36.56 & 12.47 & 27.63 & 23.49 & \\
& $36.5 \%$ & $12.5 \%$ & $27.6 \%$ & $23.5 \%$ & 0.070 \\
100 & 133.16 & 496.22 & 606.36 & 189.68 & \\
& $9.3 \%$ & $34.8 \%$ & $42.5 \%$ & $13.3 \%$ & 0.137 \\
101 & 6.77 & 21.01 & 36.11 & 11.78 & \\
& $8.9 \%$ & $27.8 \%$ & $47.7 \%$ & $15.6 \%$ & 0.127 \\
110 & 13.71 & 18.65 & 14.47 & 26.35 & \\
& $18.7 \%$ & $25.5 \%$ & $19.8 \%$ & $36.0 \%$ & 0.057 \\
111 & 247.86 & 151.55 & 373.07 & 419.48 & \\
& $20.8 \%$ & $12.7 \%$ & $31.3 \%$ & $35.2 \%$ & 0.082 \\
\hline
\end{tabular}

Upper figures in the cells represent the sum total of the acquired weight in all the agents, and lower figures are the acquired weight ratio in the condition rule type. AVEDEV is average deviation of the weight values.

Since work agent can detect the direction towards the target process machine, the weight values of condition rules in ' 000 ', ' 100 ' and ' 111 ' naturally become large in the tables. We can observe several interesting reinforcement phenomena, which conducts better scheduling performance, in Type B (Table 3 (a)) as follows:

i) Rule with Action part ' 00 ' is reinforced in Condition Part ' 000 '

ii) Rules with Action part ' 01 ' and ' 10 ' are reinforced instead of the one with Action Part ' 00 ', in Condition Part ' 100 '

iii) Rules with Action part ' 11 ' and ' 10 ' are reinforced instead of the one with Action Part ' 00 ', in Condition Part ' 111 '

Item i) is naturally understandable to acquire smooth movement in work agents. There always exist agent groups around the process machines, and it is important to keep the balance in agent density for the efficiency and the robustness in the proposed reactive scheduling. Item ii) and iii) are deeply concerned with the 
balanced density of our work agent. Rule with Action part ' 10 ' leads the temporal evacuation movement of agents from dense populated area. It is not always efficient to go back in short-term aspect, but sensible in long term strategy. Rule with Action part '01' in Condition Part '100' and Rule with Action part '11' in Condition Part ' 111 ' leads the rational movement in the collision between any two agents. Two collided work agents naturally face the opposite direction to avoid the consecutive collisions by those rules. Reward / penalty values are moderate and well-balanced agent strategy is acquired in Type B.

Whereas reward / penalty values are too large to acquire well-balanced agent strategy in Type C. Because agent learning strongly depends on its initial conditions, acquired weight patterns are ill converged in Table 3 (b).

\subsection{Cooperation algorithm and profit sharing approach}

Our cooperation algorithm is based on the biological entrainment phenomena described in 2.2. Although the entrainment algorithm is proved to be quite effective to form an self-organised work flow smoothly, the concept is not always fit the learned rules in Table 3 (a) due to the lack of step back motion. Therefore it is quite interesting to investigate the relation between the cooperation algorithm and profit sharing scheme. Simulations result without the entrainment algorithm is shown in Figure 5. Cooperative motion is purely conducted by the learned weight of rules.

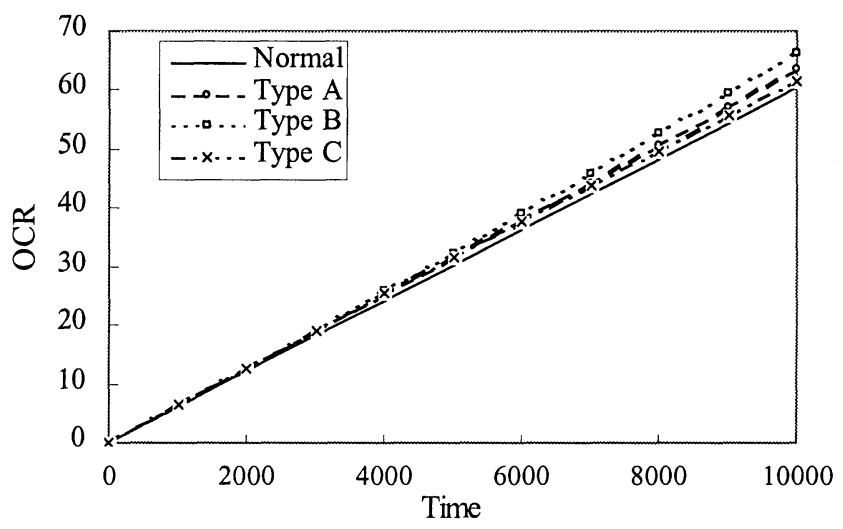

Figure 5 Scheduling performance without entrainment

Schedule performance in profit sharing is better than the conventional selforganised scheduling in any experimental conditions. In our previous research, it has been proved that 'the entrainment algorithm is robust enough to adapt practical use, but the algorithm doesn't always conduct the optimal scheduling solutions'. Acquired rule set with step back motion by profit sharing overcomes the drawback and has been proved effective in the reactive self-organised scheduling. 


\section{CONCLUSIONS}

In this paper a reactive self-organised scheduling using cooperative autonomous agent with learning algorithm is proposed. The architecture is quite simple but suitable to fully de-centralised scheduling system. Profit sharing naturally creates the step back motion in agents so as to keep moderate population density around the process machines for their smooth movement. The conventional entrainment algorithm is not always compatible with the profit sharing scheme. The effectiveness of the proposed methodology has been investigated and welleducated work agents have proved capable of robustness and reliability coping with the several scheduling demands.

\section{REFERENCES}

Colorni, A., et al (1992) Distributed Optimization by ant colonies, Proceedings of the First European Conference on Artificial Life, The MIT Press.

Deneubourg, J. (1991) The dynamics of collective sorting robot-like ants and antlike robots, Proc. of the First Int. Conference on Simulation of Adaptive Behavior, The MIT Press.

Gredenstette J. J. (1988) Credit Assignment in Rule Discovery Systems Based on Genetic Algorithms, Machine Learning, 3, 225-245.

Kaihara T. and Fujii S. (1997) A self-organization scheduling paradigm using coordinated autonomous agents, Rapid Product Development, Chapman \& Hall, London, 489-498.

Kaihara T. and Fujii S. (1998) An Evolutionary Scheduling Paradigm using Coordinated Autonomous Agents, Innovation, Globalization of Manufacturing in the Digital Communication Era of the $21^{\text {st }}$ Century, Kluwer Academic Publishers, Boston, 553-563.

Langton C.G. (1986) Studying artificial life with cellular automata, Physica D, Vol.22, 120-149.

Uzsoy R., Lee C. and Martin-Vega L.A. (1992) A Review of production planning and scheduling in the semiconductor industry, IIE Transactions, Vol.24, No.4, 47-57.

\section{BIOGRAPHY}

Toshiya Kaihara received the B.E. and M.E. degrees in precision engineering from Kyoto University, Kyoto, Japan, and Ph.D. degree in mechanical engineering from Imperial College, University of London, London, UK. He worked for Mitsubishi Electric Corp. and joined University of Marketing and Distribution Sciences as an associate professor in 1996. His research interests include multi agent-based modelling of manufacturing / distribution system and supply chain management.

He is a member of ISCIE, IEEJ, IFIP, IEEE, and others. 
Susumu Fujii is a Professor of Computer and Systems Engineering at Kobe University, Kobe, Japan. He received a B.S. in Mechanical Engineering and M.S. in Precision Engineering from Kyoto University and Ph.D. in Mechanical Engineering from the University of Wisconsin, Madison. His research interests include modelling and analysis of computer integrated manufacturing systems, production planning and scheduling, and manufacturing system simulation.

$\mathrm{He}$ is a member of various academic societies such as JSPE, JSME, ORSJ, ISCIE, SICE, INFORMS, IFIP and others.

Satoshi Kunimasa is a post graduate student in Kobe University, Kobe, Japan. He received a B.E. in Computer and System Engineering in Kobe University in 1997. His research interests include multi-agent programming and manufacturing scheduling. 